\title{
Article \\ Catalase Protects Biofilm of Staphylococcus aureus against Daptomycin Activity
}

\author{
Cristina El Haj ${ }^{1}$, Mads Lichtenberg ${ }^{1}{ }^{\mathbb{D}}$, Karen Leth Nielsen ${ }^{2}$, Thomas Bjarnsholt ${ }^{1,2}$ and Peter $Ø$ strup Jensen ${ }^{1,2,3, *}$ \\ 1 Costerton Biofilm Center, Department of Immunology and Microbiology, Faculty of Health Sciences, \\ University of Copenhagen, DK-2200 Copenhagen, Denmark; celhaj6@gmail.com (C.E.H.); \\ mlichtenberg@sund.ku.dk (M.L.); tbjarnsholt@sund.ku.dk (T.B.) \\ 2 Department of Clinical Microbiology, Rigshospitalet, DK-2200 Copenhagen, Denmark; \\ karen.leth.nielsen.01@regionh.dk \\ 3 Institute for Inflammation Research, Center for Rheumatology and Spine Diseases, Rigshospitalet, \\ DK-2200 Copenhagen, Denmark \\ * Correspondence: peter.oestrup.jensen@regionh.dk
}

Citation: El Haj, C.; Lichtenberg, M.; Nielsen, K.L.; Bjarnsholt, T.; Jensen, P.Ø. Catalase Protects Biofilm of Staphylococcus aureus against Daptomycin Activity. Antibiotics 2021, 10, 511. https://doi.org/10.3390/ antibiotics 10050511

Academic Editor:

Malgorzata Paduszynska

Received: 18 March 2021

Accepted: 27 April 2021

Published: 30 April 2021

Publisher's Note: MDPI stays neutral with regard to jurisdictional claims in published maps and institutional affiliations.

Copyright: () 2021 by the authors. Licensee MDPI, Basel, Switzerland. This article is an open access article distributed under the terms and conditions of the Creative Commons Attribution (CC BY) license (https:// creativecommons.org/licenses/by/ $4.0 /)$.

\begin{abstract}
Daptomycin is recommended for the treatment of Staphylococcus aureus infections due to its bactericidal activity. However, its mechanism of action is poorly understood. The involvement of reactive oxygen species (ROS) in the bactericidal activity of daptomycin has been proved against planktonic S. aureus, but not against the biofilm of S. aureus. Therefore, we evaluated if ROS contributes to the effect of daptomycin against biofilm of $S$. aureus. Biofilms of wild type, catalase deficient and daptomycin-resistant $S$. aureus strains were grown in microtiter-plates. After three days, the biofilms were exposed to daptomycin with or without thiourea in the presence of a ROS indicator. After overnight incubation, the amount of ROS and the percentage of surviving bacteria were determined. The bacterial survival was higher and the amount of ROS was lower in the wild type than in the catalase deficient biofilm, demonstrating a protective effect of catalase against daptomycin. The induction of cytotoxic ROS formation by daptomycin was verified by the addition of thiourea, which reduced the amount of ROS and protected the wild type biofilm against high concentrations of daptomycin. Accordingly, only the highest concentration of daptomycin reduced the bacterial survival and increased the ROS formation in the resistant biofilm. In conclusion, daptomycin induced the production of cytotoxic levels of endogenous ROS in S. aureus biofilm and the presence of catalase protected the biofilm against the lethality of the induced ROS.
\end{abstract}

Keywords: biofilm; Staphylococcus aureus; daptomycin; catalase

\section{Introduction}

Biofilm-related infections have become a major clinical challenge. Bacterial biofilmrelated infections exhibit tolerance to antibiotics and are protected against the host responses, making it extremely difficult to treat and eradicate infectious biofilm [1,2]. Staphylococcus aureus is one of the main microorganisms involved in biofilm-related human infections and its ability to develop antimicrobial resistance adds even more complexity to the treatment of these infections [3].

In recognition of these challenges, many new anti-biofilm therapeutic strategies are being investigated. The strategies are focussed on the eradication of biofilm-embedded bacteria, which present different metabolic conditions than those in the planktonic state of growth. In recent years, peptides have appeared as a novel approach to anti-biofilm treatment, as exemplified by using colistin to treat biofilms formed by Gram-negative bacilli. It has been suggested that colistin may be highly effective against dormant bacteria in anaerobic conditions, since colistin's anti-biofilm activity is not dependent on the production of hydroxyl radicals [4]. 
Among the particular pipeline of anti-staphylococcal antibiotics, daptomycin has attracted attention due to its activity against growing and non-growing staphylococcal bacteria and daptomycin is effective against biofilm-related infections [5-7]. The mechanism of action of daptomycin is, however, not well understood [8,9], but the bactericidal effect of daptomycin against planktonic bacteria may rely on the endogenous formation of bactericidal reactive oxygen species (ROS) [10].

With this in mind, we examined if daptomycin induces the formation of toxic ROS in S. aureus biofilm by estimating the effect of catalase and the effect of ROS scavenging by thiourea. The rationale for our experimental approach is outlined in Figure 1.

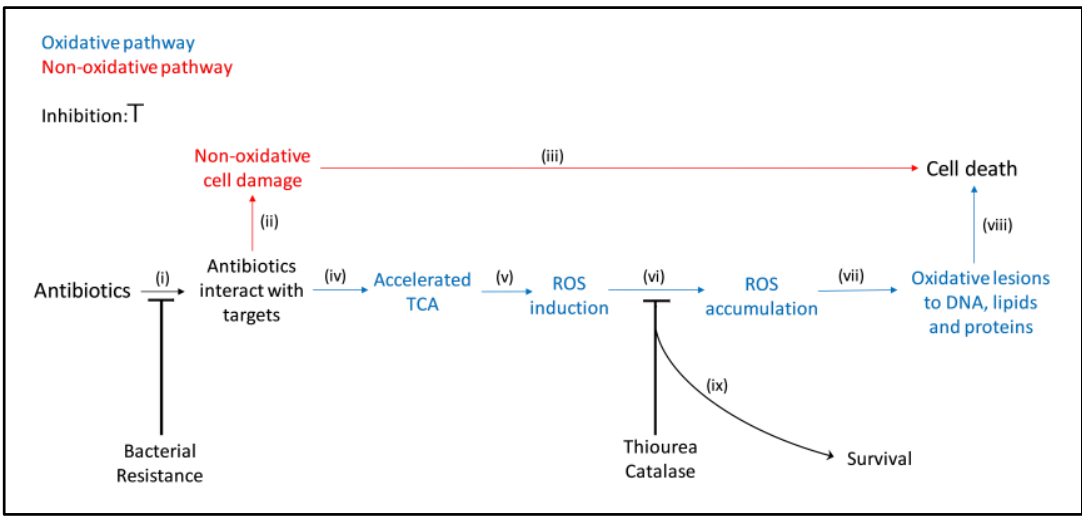

Figure 1. Schematic mechanisms of the main steps involved in the bactericidal activity of antibiotics. When antibiotics interact with their targets, (i) primary damage may occur. If this primary damage is severe and independent of oxidative stress, (ii) bacteria are not able to repair the damage (iii) and die (red). If the primary damage is insufficient to the kill the bacteria directly, the tricarboxylic acid cycle (TCA) (iv) may be accelerated, leading to the formation of ROS (v). Accumulation of ROS (vi) may cause secondary damage by introducing oxidative lesions in DNA, lipids and proteins (vii), resulting in killing of the bacteria (viii). Modified from Kohanski et al., 2007, Zhao et al., 2015, Van Acker and Coenye, 2017 [11-13]. In this study, the involvement of the oxidative pathway in the bactericidal activity of daptomycin was evaluated by the increased survival (ix) and the decreased ROS formation when ROS was removed with catalase or scavenged with thiourea (vi) and by preventing interactions with the targets in a resistant isolate (i).

\section{Results}

\subsection{Catalase Protects S. aureus Biofilm against Daptomycin}

The contribution of ROS to the bactericidal activity of antibiotics is controversial [11,14-16], but examining susceptibility in mutants with deficient anti-oxidative mechanisms has long been recognized as a valid approach to estimate cytotoxic contributions of ROS to the activity of antibiotics [17]. Therefore, we compared the effect of catalase on daptomycin treatment using the wild type S. aureus strain (SH1000) and a S. aureus strain with deficient catalase activity $(\mathrm{KO})$. We found that catalase deficiency was associated with significantly decreased survival during daptomycin treatment, demonstrating that catalase protects biofilm of S. aureus against daptomycin (Figure 2). The deficient catalase activity of $\mathrm{KO}$ was verified by the increased susceptibility to hydrogen peroxidase $\left(\mathrm{H}_{2} \mathrm{O}_{2}\right)$ (Figure S1). A further indication of the involvement of ROS in the bactericidal activity of daptomycin on biofilm was demonstrated by the indicator of ROS, $2^{\prime}, 7^{\prime}$-dicholorodihydrofluorescein diacetate (DCHF), which was only enhanced significantly at daptomycin concentrations that significantly reduced survival in SH1000 and in KO biofilm (Figure S2). Altogether, these results indicate that catalase protects $S$. aureus biofilm against cytotoxic ROS induced by daptomycin. 


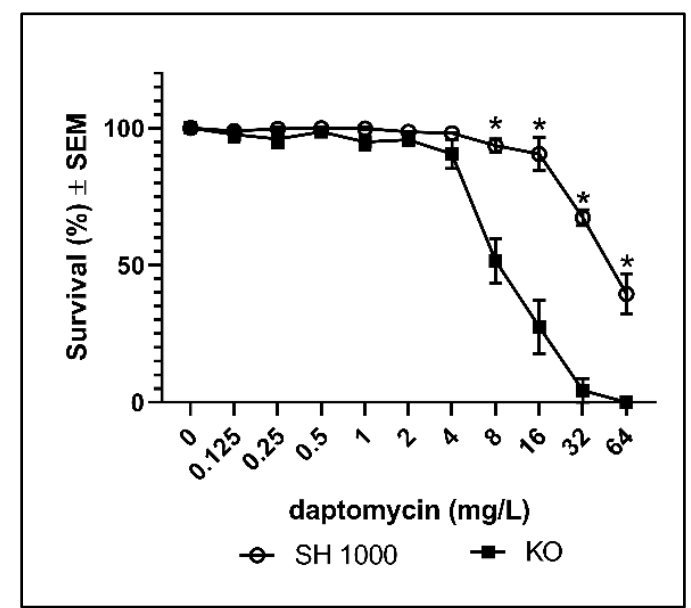

Figure 2. Percentages surviving cells demonstrating the protective effect of catalase against daptomycin treatment of S. aureus biofilms. Biofilms of the wild type (SH1000) and of the catalase deficient strain $(\mathrm{KO})$ were grown for 3 days before overnight treatment with daptomycin. Mean \pm SEM of triplicate experimental set-ups are shown. Statistical significance was determined using two-way ANOVA, followed by Bonferroni's multiple comparison tests. ${ }^{*} p<0.05$.

\subsection{Thiourea Protects S. aureus Biofilm against Daptomycin}

To validate the contribution of ROS to the bactericidal activity of daptomycin, we added thiourea during treatment with daptomycin. Thiourea is a specific scavenger of hydroxyl radicals [18], which is a ROS that may contribute to the bactericidal activity against planktonic and biofilm bacteria $[11,19]$. The addition of thiourea to SH1000 biofilm significantly increased survival during daptomycin treatment (Figure 3a) and the DCHF fluorescence was significantly reduced (Figure $3 b$ ). Thus, the addition of thiourea further demonstrated that ROS might contribute to the activity of daptomycin on S. aureus biofilm.

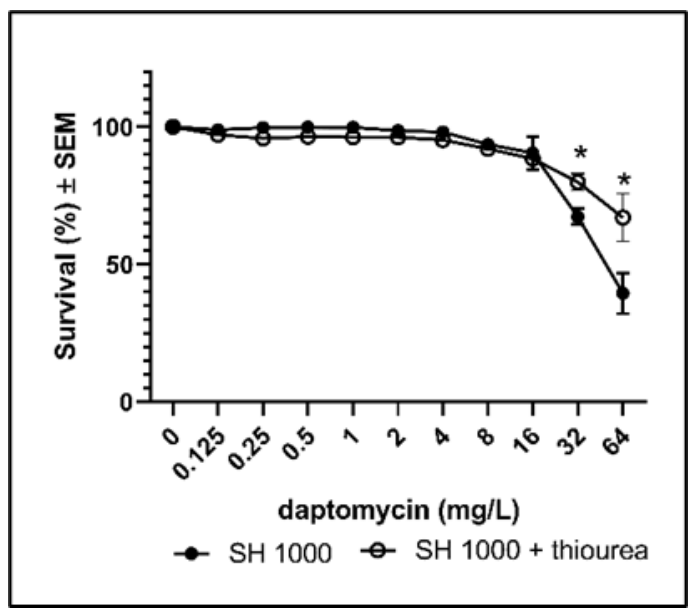

(a)

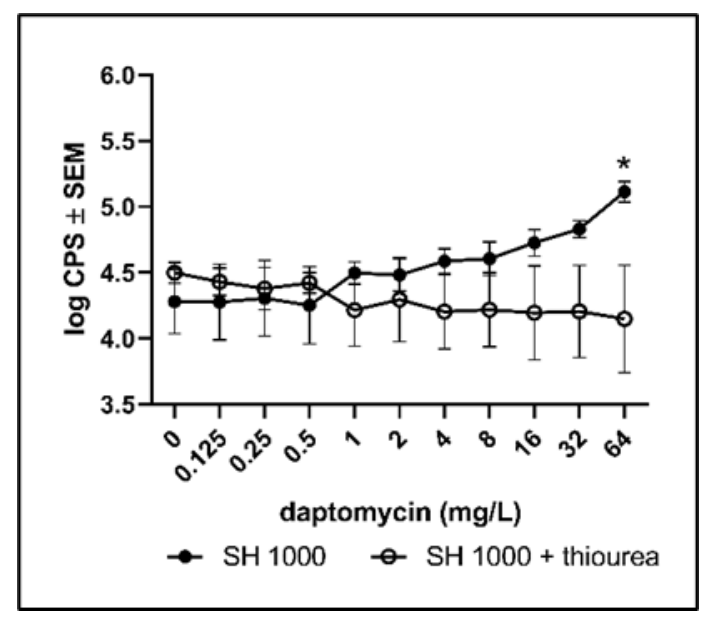

(b)

Figure 3. The rescuing effect of scavenging ROS with thiourea. Biofilms of the wild type (SH1000) were grown for 3 days before assessing the effect of the addition of thiourea during overnight treatment with daptomycin. (a) Bacterial survival was estimated by plating and counting of CFUs. (b) ROS formation according to DCHF fluorescence. Mean \pm SEM of triplicate experimental set-ups are shown. Statistical significance was determined using two-way ANOVA, followed by Bonferroni's multiple comparison tests. ${ }^{*} p<0.05$. 


\subsection{Reduced Survival and Increased ROS Accumulation in Biofilm of a Resistant Clinical Isolate} Requires High Concentrations of Daptomycin

To examine if biofilm of resistant clinical isolates may avoid the induction of cytotoxic ROS by daptomycin, as part of their protective strategy, we exposed biofilm of a resistant clinical isolate, HUB512, to daptomycin. Daptomycin was only able to reduce the survival and increase the DCHF fluorescence significantly at the highest concentration $(64 \mathrm{mg} / \mathrm{L})$ (Figure 4). This indicates that increased tolerance against daptomycin in S. aureus biofilm is associated with a decreased amount of ROS induction. Whole-genome sequencing data revealed a mutation, T345I, in $m p r F$, which has previously been identified as a cause of lower susceptibility towards daptomycin [20,21].

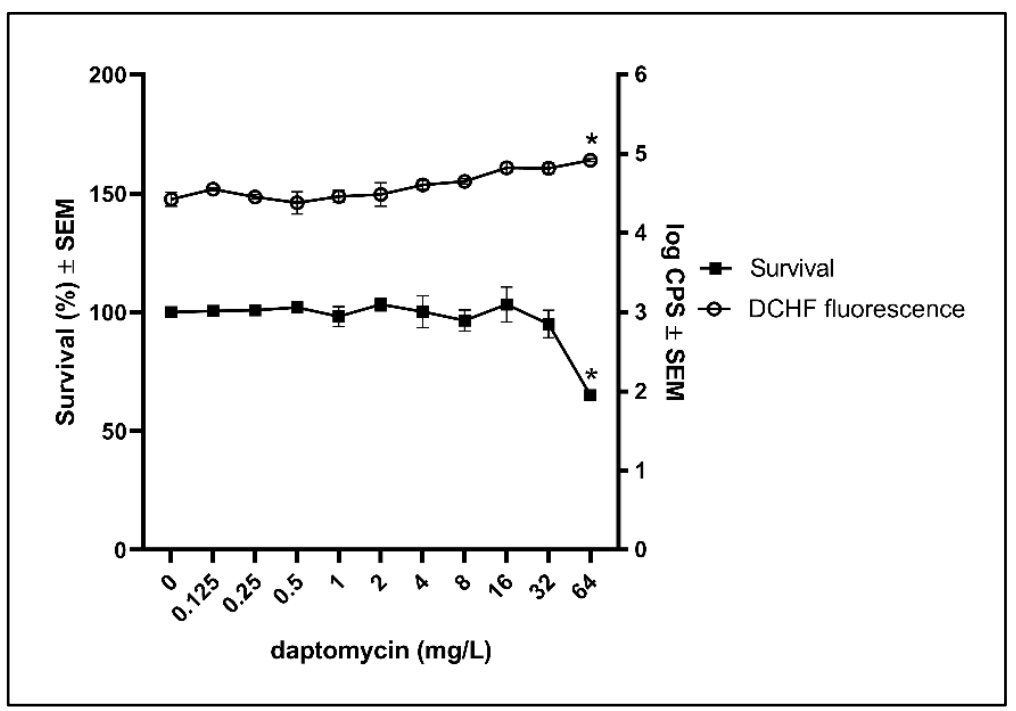

Figure 4. Effect of daptomycin on bacterial survival and the DCHF fluorescence in biofilm of a resistant clinical isolate. Biofilms of resistant clinical isolate (HUB512) were grown for 3 days before estimating the effect of daptomycin on the survival and ROS formation according to DCHF. Mean \pm SEM of triplicate experimental set-ups are shown. Statistical significance was determined using one-way ANOVA, followed by Bonferroni's multiple comparison tests. ${ }^{*} p<0.05$.

\section{Discussion}

In this study, catalase and thiourea were able to protect three-day-old biofilm of S. aureus against daptomycin, indicating that ROS may contribute to the bactericidal activity of daptomycin on S. aureus biofilm. ROS may be involved in the bactericidal activity of aminoglycosides, fluoroquinolones and beta-lactams against planktonic bacteria [12]. In biofilm-embedded bacteria, a contribution of ROS to the antimicrobial activity of ciprofloxacin was demonstrated in biofilms of Pseudomonas aeruginosa [19]. However, the bactericidal activity of antimicrobial peptides may not depend on endogenous ROS, as in the case of colistin acting on planktonic cultures and the biofilm of P. aeruginosa $[4,16]$.

The lipopeptide daptomycin has bactericidal activity against $S$. aureus in $\log$ and the stationary-phase of growth and has anti-biofilm activity against staphylococcal biofilm [22]. Daptomycin acts by disrupting cell membranes and killing by daptomycin does not require cell division or active metabolism. Daptomycin binds to calcium and integrates into the cytoplasmatic membrane where daptomycin forms ion-permeable transmembrane channels, causing changes in the membrane potential leading to cell rupture [23]. In contrast to other anti-staphylococcal antibiotics, daptomycin rapidly penetrates staphylococcal biofilms [6]. In addition, the frequency of development of daptomycin resistance is low, though strains with reduced susceptibility to daptomycin have been found in clinical practice [5]. Thus, current recommendations for the treatment of difficult infections encourage the use of daptomycin at high concentrations $(8-10 \mathrm{mg} / \mathrm{kg} /$ day), in combination with other antibiotics [24]. 
Since daptomycin is a peptide-like antibiotic, it might be expected that the bactericidal effect of daptomycin resembles the mechanism of action of colistin on P. aeruginosa, which does not require endogenous ROS [4,16]. Nevertheless, Liu et al. [10] demonstrated a correlation between the activity of daptomycin and the formation of ROS in planktonic S. aureus. However, the contribution of ROS to the bactericidal effect of daptomycin has not been studied before against staphylococcal biofilm.

Compared to the wild type strain, the catalase deficient strain showed lower survival when treated with daptomycin, indicating that the wild type strain is more efficiently protected against endogenous $\mathrm{H}_{2} \mathrm{O}_{2}$. According to Kohanski et al. [11], $\mathrm{H}_{2} \mathrm{O}_{2}$ is a precursor for hydroxyl radicals, which are bactericidal and can be neutralized by the specific scavenger thiourea $[18,25]$. Therefore, the ability of thiourea to reduce the ROS indicator and increase the survival in the wild type biofilm treated with daptomycin is further confirming a contribution of ROS to the bactericidal effect of daptomycin on S. aureus biofilm. It may be argued that the protection of thiourea is derived from the induction of slow growth [17]. In our study, however, we treated it with $100 \mathrm{mM}$ of thiourea, which did not affect growth (Supplementary Figure S3).

Estimation of ROS formation in biofilm may be achieved by using DCHF as an indicator for ROS [19]. In addition, treatments with concentrations of daptomycin resulting in decreased survival were accompanied with increased DCHF fluorescence in the wild type and in the catalase deficient mutant, which further emphasizes the contribution of endogenous ROS to the bactericidal activity of daptomycin on $S$. aureus biofilm. The decreased DCHF fluorescence during the rescuing of the wild type from daptomycin with thiourea also supports that endogenous ROS contributes to the bactericidal activity of daptomycin.

To further verify that accumulation of cytotoxic ROS is related to interactions between antibiotics and their targets, we treated a resistant clinical isolate with daptomycin. As expected, more daptomycin $(64 \mathrm{mg} / \mathrm{L})$ was needed to detect a significant antimicrobial effect on biofilm of the resistant isolate compared to the wildtype ( $32 \mathrm{mg} / \mathrm{L}$ ). Accordingly, the DCHF fluorescence indicates the accumulation ROS in the resistant isolate was only increased at the bactericidal concentration of daptomycin. Since the resistant clinical isolate was more susceptible to $\mathrm{H}_{2} \mathrm{O}_{2}$ than the wild type (Figure S1), our results suggest the protective mechanisms of the resistant clinical isolate does not include enhanced anti-oxidative defence. Instead, we speculate, that the resistant clinical isolate disables the possibility of daptomycin to interact with its targets, which may lead to ROS formation [11]. The amino acid substitution in the mprF gene that we found in the HUB512 strain has previously been demonstrated in other isolates $[20,21]$. The mutations in the mprF gene result in increased net positive charge in the bacterial cell surface causing the repulsion of the positively charged daptomycin molecules [20].

Our results suggest that the stress on the membrane caused by daptomycin in S. aureus biofilm triggers the generation of cytotoxic ROS, as previously proposed [11]. Accordingly, interaction of daptomycin with its targets induces an increased activity of the TCA leading to super-oxidation of NADH by the enhancement of the electron transport chain, which results in superoxide formation. Subsequent dismutation of superoxide results in the production of $\mathrm{H}_{2} \mathrm{O}_{2}$, which may react with $\mathrm{Fe}^{2+}$ by Fenton reactions to produce hydroxyl radicals that is cytotoxic to $S$. aureus [25].

\section{Conclusions}

In conclusion, daptomycin activity against $S$. aureus biofilm depends on induction of ROS formation, as evidenced by the protection of the $S$. aureus biofilm against lethal oxidative stress provided by the presence of catalase and thiourea. Further studies are warranted in order to obtain a comprehensive in-depth identification of the species of ROS induced by daptomycin and the anti-oxidative mechanisms involved in the protection of S. aureus biofilm. 


\section{Materials and Methods}

\subsection{Strains and Susceptibility Studies}

In this study, we employed the SH1000 wild type, the dual katA/ahpC knockout SH1000 S. aureus strains (KO) from University of Sheffield [26] and a daptomycin resistant S. aureus isolate (HUB512) from an osteoarticular infection at Bellvitge University Hospital (Barcelona, Spain). The dual katA/ahpC mutations disables the capacity of the strain to scavenge exogenous or endogenously produced $\mathrm{H}_{2} \mathrm{O}_{2}[26,27]$.

Minimal inhibitory concentrations (MIC) for daptomycin (Cubicin, Barcelona, Spain) were determined following standard recommendations [28] to classify the strains as susceptible or resistant to daptomycin according to EUCAST breakpoints and $50 \mathrm{mg} / \mathrm{L}$ of calcium were added to media. MIC for daptomycin was $0.5 \mathrm{mg} / \mathrm{L}$ for the wild type, $1 \mathrm{mg} / \mathrm{L}$ for $\mathrm{KO}$ and $4 \mathrm{mg} / \mathrm{L}$ for the HUB512 strain.

To verify the deficient catalase activity of the $\mathrm{KO}$ strain, the susceptibility of each strain to $\mathrm{H}_{2} \mathrm{O}_{2}$ was determined. Inoculums from overnight culture were adjusted to $10^{6} \mathrm{CFU} / \mathrm{mL}$ in Mueller Hinton Broth (Becton Dickinson, Kongens Lyngby, Denmark) in a 96 well plate, exposed to $\mathrm{H}_{2} \mathrm{O}_{2}$ at concentrations of $0,10,15,20,25,30$ and $60 \mathrm{mM}$ of $\mathrm{H}_{2} \mathrm{O}_{2}$ and incubated in the dark at $37^{\circ} \mathrm{C}$ for $1 \mathrm{~h}$. Bacterial counts were determined before and after exposure by serial dilution in saline and plated on trypticase soy agar with $5 \%$ sheep blood (Becton Dickinson, Kongens Lyngby, Denmark) (Figure S1).

To determine the highest concentration of thiourea that does not inhibit growth, an inoculum of $10^{6} \mathrm{CFU} / \mathrm{mL}$ of each strain was exposed to 0,50,100, 150 and $200 \mathrm{mM}$ of thiourea (Sigma, T8656) and incubated at $37^{\circ} \mathrm{C}$ overnight. The growth was estimated by the optical density at $600 \mathrm{~nm}$ (Figure S3). Accordingly, it was decided to treat the biofilm with $100 \mathrm{mM}$ thiourea.

\subsection{Studies of Survival and Reactive Oxygen Species}

The methodology used was a modification of a previously described method [19]. Briefly, an overnight culture of each strain was resuspended in Mueller Hinton Broth supplemented with $0.5 \%$ of glucose $(w / v)$ (MHBG) to obtain an inoculum of $10^{6} \mathrm{CFU} / \mathrm{mL}$. The final inoculum was added to each well of black microtiter plates with transparent flat bottom (Thermos Fisher scientific, New York, NY, USA) and incubated at $37^{\circ} \mathrm{C}$. After 3 days, the supernatant was replaced by $200 \mu \mathrm{L}$ of daptomycin solution in MHBG in twofold dilution concentrations from 0 to $64 \mathrm{mg} / \mathrm{L}$ and $50 \mathrm{mg} / \mathrm{L}$ of calcium (with or without $100 \mathrm{mM}$ of thiourea). To detect a broad range of ROS, $2^{\prime}, 7^{\prime}$-dicholorodihydrofluorescein diacetate (DCHF-DA; Sigma, Copenhagen, Denmark) was added to each well at a final concentration of $5 \mu \mathrm{M}$. Then, the plates were incubated in the dark at $37^{\circ} \mathrm{C}$ overnight.

After incubation, the fluorescence of the biofilms was recorded at excitation/emission wavelength $485 / 535 \mathrm{~nm}$ on a plate-reader (Wallac 1420, Victor X2, Perkin Elmer, Boston, MA, USA). To eliminate the influence of autofluorescence caused by daptomycin, the fluorescence from treated cultures without DCHF-DA was subtracted. Surviving bacteria were determined by bacterial counts with a limit of detection of $10 \mathrm{CFU} / \mathrm{mL}$.

All experiments were performed as biological triplicates.

\subsection{Whole-Genome Sequencing}

DNA of clinical isolate HUB512 was extracted using DNeasy blood and Tissue kit (Qiagen, Hilden, Germany), and libraries were created with Nextera XT (Illumina, San Diego, CA, USA). The isolate was sequenced on a NextSeq (Illumina) using paired-end technology $(2 \times 250 \mathrm{bp})$. Single nucleotide polymorphisms were identified using BacDist with S. aureus GCF_000013425.1 [29]. Analyses of BacDist output was performed in order to identify known polymorphisms in relevant genes causing daptomycin resistance (based on The Comprehensive Antibiotic Resistance Database (https: / / card.mcmaster.ca, accessed on 31 October 2020)). 


\subsection{Statistical Analyses}

Data were analysed using Prism 8.0 (GraphPad Software Inc., La Jolla, CA, USA). All bacterial counts were represented as a percentage of surviving cells (mean $\pm \mathrm{SEM}$ ), taking bacterial counts from untreated control as a percentage equivalent to $100 \%$. Comparisons of strains and effects of the addition of thiourea were performed by two-way ANOVA. The effects of daptomycin treatment on the percentage of surviving cells and the fluorescence of DCHF of each strain were evaluated by one-way analysis of variance (ANOVA). Adjustment for multiple comparisons was done by Bonferroni's multiple comparison correction. Differences were considered statistically significant at $p<0.05$.

Supplementary Materials: The following are available online at https:/ /www.mdpi.com/article/10 .3390/antibiotics10050511/s1, Figure S1: Susceptibility of SH1000, catalase deficient and daptomycin resistant S. aureus strains to hydrogen peroxide, Figure S2: Survival and DCHF fluorescence in biofilms of SH1000 (a) and KO (b) treated with daptomycin, Figure S3: Percentage of bacterial survival with thiourea.

Author Contributions: C.E.H. and P.Ø.J. designed the study, C.E.H. and K.L.N. performed the experiments; C.E.H., K.L.N. and P.Ø.J. analyzed results; C.E.H., M.L., K.L.N., T.B. and P.Ø.J. interpreted data, have drafted the work and substantively revised it. All authors have read and agreed to the published version of the manuscript.

Funding: C.E. was funded by the Martin Luengo 2019 grant awarded by the Spanish Society of Infectious Diseases and Clinical Microbiology (SEIMC).

Data Availability Statement: The data that support the findings of this study are available on request from the corresponding author. The data are not publicly available due to privacy or ethical restrictions. The raw reads from the sequencing are deposited in SRA under accession PRJNA725634.

Acknowledgments: The authors thank Andrew M. Edwards (Imperial College London), Simon J. Foster (University of Sheffield) and Fe Tubau (Bellvitge University Hospital, Barcelona) for providing us the strains used in this study. We also wish would thank Yanik Sierra (Bellvitge University Hospital, Barcelona) for his help in screening of resistance-associated mutations. C.E. is grateful to the Martin Luengo fellowship (SEIMC) for making this research possible.

Conflicts of Interest: The authors declare no conflict of interest.

\section{References}

1. Del Pozo, J.L.; Patel, R. The challenge of treating biofilm-associated bacterial infections. Clin. Pharm. Ther. 2007, 82, $204-209$. [CrossRef] [PubMed]

2. Stewart, P.S.; Costerton, J.W. Antibiotic resistance of bacteria in biofilms. Lancet 2001, 358, 135-138. [CrossRef]

3. Archer, N.K.; Mazaitis, M.J.; Costerton, J.W.; Leid, J.G.; Powers, M.E.; Shirtliff, M.E. Staphylococcus aureus biofilms: Properties, regulation, and roles in human disease. Virulence 2011, 2, 445-459. [CrossRef] [PubMed]

4. Kolpen, M.; Appeldorff, C.F.; Brandt, S.; Mousavi, N.; Kragh, K.N.; Aydogan, S.; Uppal, H.A.; Bjarnsholt, T.; Ciofu, O.; Høiby, N.; et al. Increased bactericidal activity of colistin on Pseudomonas aeruginosa biofilms in anaerobic conditions. Pathog. Dis. 2016, 74, ftv086. [CrossRef]

5. Steenbergen, J.N.; Alder, J.; Thorne, G.M.; Tally, F.P. Daptomycin: A lipopeptide antibiotic for the treatment of serious grampositive infections. J. Antimicrob. Chemother. 2005, 55, 283-288. [CrossRef]

6. Stewart, P.S.; Davison, W.M.; Steenbergen, J.N. Daptomycin rapidly penetrates a Staphylococcus epidermidis biofilm. Antimicrob. Agents Chemother. 2009, 53, 3505-3507. [CrossRef] [PubMed]

7. Rybak, M.J. The efficacy and safety of daptomycin: First in a new class of antibiotics for gram-positive bacteria. Clin. Microbiol. Infect. 2006, 12, 24-32. [CrossRef] [PubMed]

8. Heidary, M.; Khosravi, A.D.; Khoshnood, S.; Nasiri, M.J.; Soleimani, S.; Goudarzi, M. Daptomycin. J. Antimicrob. Chemother. 2018, 73, 1-11. [CrossRef] [PubMed]

9. Müller, A.; Wenzel, M.; Strahl, H.; Grein, F.; Saaki, T.N.V.; Kohl, B.; Siersma, T.; Bandow, J.E.; Sahl, H.G.; Schneider, T.; et al. Daptomycin inhibits cell envelope synthesis by interfering with fluid membrane microdomains. Proc. Natl. Acad. Sci. USA 2016, 113, 7077-7086. [CrossRef]

10. Liu, Y.; Liu, X.; Qu, Y.; Wang, X.; Li, L.; Zhao, X. Inhibitors of reactive oxygen species accumulation delay and/or reduce the lethality of several antistaphylococcal agents. Antimicrob. Agents Chemother. 2012, 56, 6048-6050. [CrossRef]

11. Kohanski, M.A.; Dwyer, D.J.; Hayete, B.; Lawrence, C.A.; Collins, J.J. A common mechanism of cellular death induced by bactericidal antibiotics. Cell 2007, 130, 797-810. [CrossRef] [PubMed] 
12. Zhao, X.; Hong, Y.; Drlica, K. Moving forward with reactive oxygen species involvement in antimicrobial lethality. J. Antimicrob. Chemother. 2015, 70, 639-642. [CrossRef]

13. Van, A.H.; Coenye, T. The role of reactive oxygen species in antibiotic-mediated killing of bacteria. Trends Microbiol. 2017, 25, 456-466. [CrossRef]

14. Keren, I.; Wu, Y.; Inocencio, J.; Mulcahy, L.R.; Lewis, K. Killing by bactericidal antibiotics does not depend on reactive oxygen species. Science 2013, 339, 1213-1216. [CrossRef]

15. Liu, Y.; Imlay, J.A. Cell death from antibiotics without the involvement of reactive oxygen species. Science 2013, 339, 1210-1213. [CrossRef]

16. Brochmann, R.P.; Toft, A.; Ciofu, O.; Briales, A.; Kolpen, M.; Hempel, C.; Bjarnsholt, T.; Høiby, N.; Jensen, P.Ø. Bactericidal effect of colistin on planktonic Pseudomonas aeruginosa is independent of hydroxyl radical formation. Int. J. Antimicrob. Agents 2014, 43, 140-147. [CrossRef] [PubMed]

17. Hassett, D.J.; Imlay, J.A. Bactericidal antibiotics and oxidative stress: A radical proposal. ACS Chem. Biol. 2007, 2, 708-710. [CrossRef]

18. Wasil, M.; Halliwell, B.; Grootveld, M.; Moorhouse, C.P.; Hutchison, D.C.; Baum, H. The specificity of thiourea, dimethylthiourea and dimethyl sulphoxide as scavengers of hydroxyl radicals. Their protection of alpha 1-antiproteinase against inactivation by hypochlorous acid. Biochem. J. 1987, 243, 867-870. [CrossRef]

19. Jensen, P.Ø.; Briales, A.; Brochmann, R.P.; Wang, H.; Kragh, K.N.; Kolpen, M.; Hempel, C.; Bjarnsholt, T.; Høiby, N.; Ciofu, O. Formation of hydroxyl radicals contributes to the bactericidal activity of ciprofloxacin against pseudomonas aeruginosa biofilms. Pathog. Dis. 2014, 70, 440-443. [CrossRef] [PubMed]

20. Nakamura, M.; Kawada, H.; Uchida, H.; Takagi, Y.; Obata, S.; Eda, R.; Hanaki, H.; Kitasato, H. Single nucleotide polymorphism leads to daptomycin resistance causing amino acid substitution-T345I in MprF of clinically isolated MRSA strains. PLoS ONE 2021, 16, e0245732. [CrossRef]

21. Tran, T.T.; Munita, J.M.; Arias, C.A. Mechanisms of drug resistance: Daptomycin resistance. Ann. N. Y. Acad. Sci. 2015, 1354, 32-53. [CrossRef]

22. Fowler, V.G.; Boucher, H.W.; Corey, G.R.; Abrutyn, E.; Karchmer, A.W.; Rupp, M.E.; Levine, D.P.; Chambers, H.F.; Tally, F.P.; Vigliani, G.A.; et al. Daptomycin versus standard therapy for bacteremia and endocarditis caused by Staphylococcus aureus. N. Engl. J. Med. 2006, 355, 653-665. [CrossRef] [PubMed]

23. Mascio, C.T.M.; Alder, J.D.; Silverman, J.A. Bactericidal action of daptomycin against stationary-phase and nondividing Staphylococcus aureus cells. Antimicrob. Agents Chemother. 2007, 51, 4255-4260. [CrossRef] [PubMed]

24. Liu, C.; Bayer, A.; Cosgrove, S.E.; Daum, R.S.; Fridkin, S.K.; Gorwitz, R.J.; Kaplan, S.L.; Karchmer, A.W.; Levine, D.P.; Murray, B.E.; et al. Clinical practice guidelines by the infectious diseases society of America for the treatment of methicillin-resistant Staphylococcus aureus infections in adults and children. Clin. Infect. Dis. 2011, 52, 18-55. [CrossRef] [PubMed]

25. Repine, J.E.; Fox, R.B.; Berger, E. Hydrogen peroxide kills Staphylococcus aureus by reacting with staphylococcal iron to form hydroxyl radical. J. Biol. Chem. 1981, 256, 7094-7096. [CrossRef]

26. Cosgrove, K.; Coutts, G.; Jonsson, I.M.; Tarkowski, A.; Kokai-Kun, J.F.; Mond, J.J.; Foster, S.J. Catalase (KatA) and alkyl hydroperoxide reductase (AhpC) have compensatory roles in peroxide stress resistance and are required for survival, persistence, and nasal colonization in Staphylococcus aureus. J. Bacteriol. 2007, 189, 1025-1035. [CrossRef]

27. Painter, K.L.; Strange, E.; Parkhill, J.; Bamford, K.B.; Armstrong-James, D.; Edwards, A.M. Staphylococcus aureus adapts to oxidative stress by producing $\mathrm{H}_{2} \mathrm{O}_{2}$-resistant small-colony variants via the SOS response. Infect. Immun. 2015, 83, 1830-1844. [CrossRef] [PubMed]

28. Patel, J.R.; Cockerill, F.R.; Bradford, P.A.; Eliopoulos, G.M.; Hindler, J.A.; Jenkins, S.G.; Lewis, J.S.; Limbargo, B.; Miller, L.A.; Nicolau, D.P.; et al. Methods for Dilution Antimicrobial Susceptibility Tests for Bacteria that Grow Aerobically, 10th ed.; Clinical and Laboratory Standards Institute: Wayne, PA, USA, 2015; p. 35.

29. GitHub MigleSur/BacDist: Snakemake Pipeline for Bacterial SNP Distance and Phylogeny Analysis. Available online: https: //github.com/MigleSur/BacDist (accessed on 24 April 2021). 